\title{
Biomechanical analysis of canine medial patellar luxation with femoral varus deformity using a computer model
}

Jiyun Lee ${ }^{1 \dagger}$, Heedong Sim ${ }^{2 \dagger}$, Jaemin Jeong ${ }^{1}$, Sun-Young Kim³ ${ }^{3}$, Seokjo Yang ${ }^{2}$, SeongMok Jeong ${ }^{1}$ and HaeBeom Lee ${ }^{1 *}$

\begin{abstract}
Background: Femoral varus deformities complicating the realignment of the quadriceps muscles are frequently associated with medial patellar luxation (MPL) in dogs. Therefore, distal femoral osteotomy (DFO) is recommended in dogs affected with severe MPL and a distal femoral varus deformity. The presence of an anatomic lateral distal femoral angle (aLDFA) of $\geq 102^{\circ}$ has been anecdotally recommended as an indication for performing corrective DFO in large-breed dogs. However, the effect of a femoral varus deformity on MPL has not been scientifically evaluated. We aimed to evaluate the influence of a femoral varus deformity on MPL using a finite element method based computer model.

Three-dimensionally reconstructed computed tomographic images of a normal femur from a Beagle dog were deformed using meshing software to create distal varus deformities. A total of thirteen aLDFAs, including $95^{\circ}, 98^{\circ}$ and $100^{\circ}-110^{\circ}$, were simulated. The patellar positions and reaction force between the patella and trochlear grooves were calculated for all finite element models under constant rectus femoris muscle activation.

Results: The patella was displaced medially from the trochlear groove at an aLDFA of $\geq 103^{\circ}$. With an aLDFA of $103^{\circ}$ to $110^{\circ}$, the reaction force was equal to zero and then decreased to negative values during the simulation, while other models with aLDFAs of $95^{\circ}, 98^{\circ}$, and $100^{\circ}-102^{\circ}$ had positive reaction force values. The patella began to luxate at 24.90 seconds (sec) with an aLDFA of $103^{\circ}, 19.80 \mathrm{sec}$ with an aLDFA of $104^{\circ}, 21.40 \mathrm{sec}$ with an aLDFA of $105^{\circ}, 20.10 \mathrm{sec}$ with an aLDFA of $106^{\circ}, 18.60 \mathrm{sec}$ with an aLDFA of $107^{\circ}, 15.30 \mathrm{sec}$ with an aLDFA of $108^{\circ}, 16.60 \mathrm{sec}$ with an aLDFA of $109^{\circ}$, and $11.90 \mathrm{sec}$ with an aLDFA of $110^{\circ}$.
\end{abstract}

Conclusion: Severe distal femoral varus with an aLDFA of $\geq 103^{\circ}$ caused MPL when other anatomical factors were controlled. Thissimplified computer model provides complementary information to anecdotal cutoffs for DFO, hence it should be applied to clinical patients with caution.

Keywords: Finite element method, Medial patellar luxation, Femoral Varus deformity, Dog

\footnotetext{
* Correspondence: seatiger76@cnu.ac.kr

${ }^{\dagger}$ Jiyun Lee and Heedong Sim contributed equally to this work.

${ }^{1}$ Department of Veterinary Surgery, College of Veterinary Medicine,

Chungnam National University, 34134 Daejeon, Republic of Korea

Full list of author information is available at the end of the article
}

(c) The Author(s). 2020 Open Access This article is licensed under a Creative Commons Attribution 4.0 International License, which permits use, sharing, adaptation, distribution and reproduction in any medium or format, as long as you give appropriate credit to the original author(s) and the source, provide a link to the Creative Commons licence, and indicate if changes were made. The images or other third party material in this article are included in the article's Creative Commons licence, unless indicated otherwise in a credit line to the material. If material is not included in the article's Creative Commons licence and your intended use is not permitted by statutory regulation or exceeds the permitted use, you will need to obtain permission directly from the copyright holder. To view a copy of this licence, visit http://creativecommons.org/licenses/by/4.0/ The Creative Commons Public Domain Dedication waiver (http://creativecommons.org/publicdomain/zero/1.0/) applies to the data made available in this article, unless otherwise stated in a credit line to the data. 


\section{Background}

Medial patellar luxation (MPL) is a common orthopedic disease of the canine stifle [1]. MPL is considered a developmental disorder because it mostly occurs after birth or early in life without trauma, although the underlying etiopathogenesis remains unclear [2, 3]. It has been suggested that coxa vara and a small anteversion angle are the underlying causes of MPL, as they lead to the medial displacement of the quadriceps femoris muscle, which results in skeletal deformities such as distal femoral varus, femoral torsion, a shallow trochlear groove, the medial displacement of the tibial tuberosity, and internal rotation of the tibia. These anatomical abnormalities of the pelvic limb have been reported as important risk factors for the malalignment of the quadriceps mechanism [1-4].

Effective surgical correction of MPL involves realignment of the quadriceps mechanism by which the quadriceps muscles contract and transfer forces to the tibia to extend the stifle joint. Thereby the patella articulates via the trochlear groove and the patellofemoral joint is stabilized [3]. The surgical procedures generally include a combination of soft tissue and bone reconstruction, such as deepening of the femoral trochlear groove and tibial tuberosity transposition $[1,5]$. The overall recurrence rate of grade 2 and $3 \mathrm{MPL}$ in dogs after surgical correction is between 6 and 11\%, while for grade $4 \mathrm{MPL}$, the recurrence rate is between 14 and $36 \%$ [5-7]. It has been reported that grade $4 \mathrm{MPL}$ in dogs is frequently associated with severe distal femoral varus deformities, thereby complicating the realignment of the quadriceps mechanism [8-10].

Distal femoral osteotomy (DFO) is a surgical technique performed to realign the quadriceps mechanism by correcting the femoral varus deformity in MPL cases $[9,11]$. Recent studies have shown good prognoses and low recurrence rates in dogs with severe MPL treated by DFO compared to previous studies in which femoral varus deformities were neither measured nor treated [5-7, 9, 11-13]. Several studies have suggested considering corrective osteotomy in small-breed dogs with significant distal femoral varus deformities $[5,8,10]$. The presence of an anatomic lateral distal femoral angle (aLDFA) of $\geq 102^{\circ}$ or a femoral varus angle (FVA) of $\geq 12^{\circ}$ has been anecdotally recommended as an indication for performing corrective DFO in large-breed dogs $[11,13]$. However, the effect of femoral varus deformities on MPL has not been scientifically evaluated. Moreover, the cutoff of aLDFA for DFO remains controversial despite the presence of anecdotal recommendations $[9,14]$.

The finite element method (FEM) is a computer-based modeling and simulation technique that has been used for the evaluation of procedures and conditions in orthopedics, such as different types of implants, surgical techniques, and pathologies [15]. In FEM, a complex geometric shape is modeled as a mesh of simpler structures (finite elements), each having appropriate biological material properties, such as the appropriate density and modulus of elasticity of muscle and bone [16]. Therefore, FEM is suitable for parametric analyses in which the effect of specific parameters are investigated in a controlled manner [16]. FEM has been widely used in human medicine as an alternative to cadaveric and in vivo biomechanical experiments [17-19]. Recently, FEM has also been increasingly frequently used in veterinary medicine $[20,21]$.

The objective of the present study was to evaluate the influence of femoral varus deformities on patellar alignment using a computer model. We hypothesized that an increase in aLDFA would increase the tendency of the MPL in the computer models.

\section{Results}

\section{Luxation of the patella}

In a simulation with the quadriceps constantly activated while stifle angle was fixed at stance phase, beginning with an aLDFA of $103^{\circ}$, the patella began to move medially and luxate (Fig. 1). In three-dimensional coordinate graphs, as aLDFA increased from $103^{\circ}$, the patella was not only displaced medially but also caudally and proximally (Fig. 2). When the aLDFA was equal to or less than $102^{\circ}$, the patella was located in the femoral trochlear groove during the simulation.

\section{Patellar dynamics according to aLDFA variation}

The reaction force (rcforce) of the $\mathrm{x}$-axis between the patella and the femoral trochlear groove was equal to zero and then decreased to negative values when the aLDFA was equal to and then exceeded $103^{\circ}$, respectively (Fig. 3). The patella began to luxate at $24.90 \mathrm{sec}$ onds (sec) with an aLDFA of $103^{\circ}, 19.80 \mathrm{sec}$ with an aLDFA of $104^{\circ}, 21.40 \mathrm{sec}$ with an aLDFA of $105^{\circ}, 20.10$ sec with an aLDFA of $106^{\circ}, 18.60$ sec with an aLDFA of $107^{\circ}, 15.30 \mathrm{sec}$ with an aLDFA of $108^{\circ}, 16.60 \mathrm{sec}$ with an aLDFA of $109^{\circ}$, and $11.90 \mathrm{sec}$ with an aLDFA of $110^{\circ}$.

\section{Discussion}

The results of this study support the initial hypothesis. In a computer model, severe distal femoral varus with an aLDFA of $\geq 103^{\circ}$ caused medial luxation of the patella when other anatomical factors of MPL were controlled. This model found complementary information to anecdotal clinical recommendations of distal femoral osteotomy to correct MPL in large-breed dogs with an aLDFA of $\geq 102^{\circ}[11,13]$. Interestingly, this is the first report to evaluate the biomechanical effect of distal femoral varus on the stability of the patella.

The biomechanics of the patellofemoral joint are complex, and standardization of the factors related to the joint 


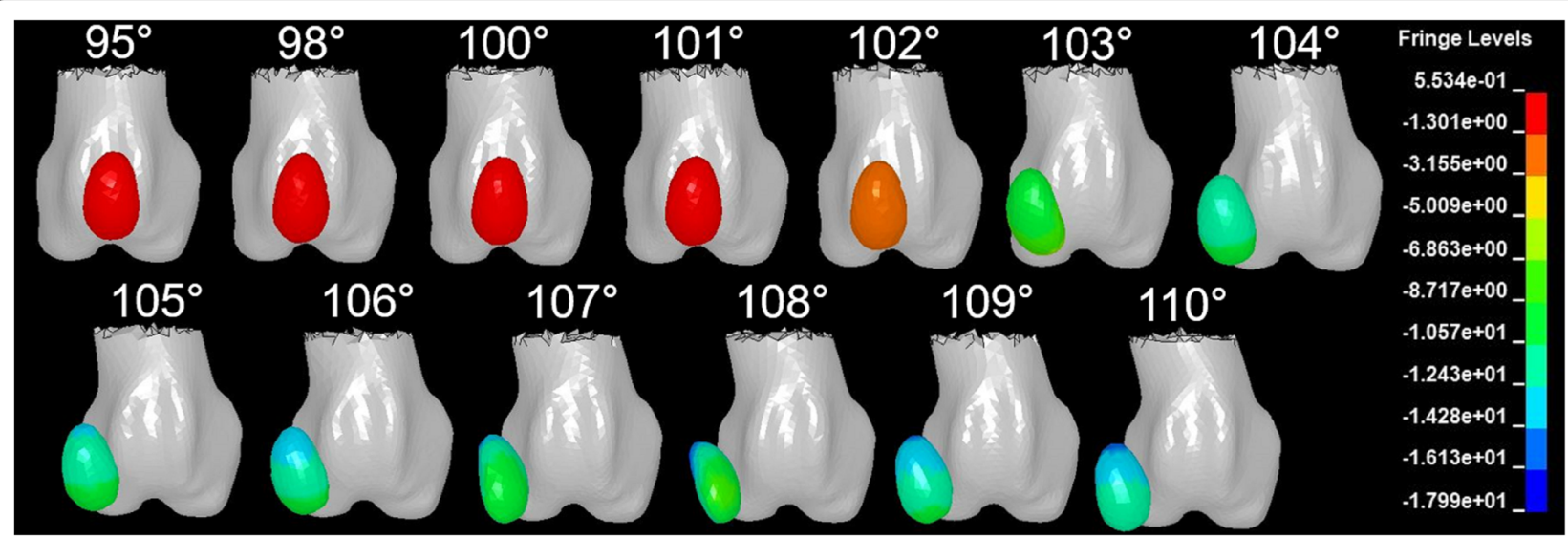

Fig. 1 The patellar position in the FE models at the last moment of constant loading on the rectus femoris muscle. The patella tended to luxate with aLDFAs equal to and greater than $103^{\circ}$ when other factors were controlled. The fringe levels indicate the magnitude of displacement of the patella from the groove, and the units of measurement are millimeters

is difficult in cadaveric and in vivo studies. Moreover, it is considered impossible to experimentally measure the whole three-dimensional state of the forces within the joint. In human medicine, FE-based computer modeling has been broadly used to investigate the biomechanics of knee joints to overcome the limitations and difficulties faced in cadaveric or in vivo studies [15, 16, 19, 22, 23]. Additionally, computer modeling is a method that avoids ethical problems related to animal use and has been increasingly used in veterinary research [20, 21, 24-26]. The current study employed computer modeling to evaluate patellar stability in the femoral trochlea with various degrees of distal femoral varus while controlling factors such as the trochlear groove shape, medial displacement of the tibial tuberosity, and internal rotation of the tibia.

The reaction force $(\mathrm{kN})$ was calculated when the patella contacted the trochlea of the femur. When the
aLDFA was larger than $103^{\circ}$, the reaction force became zero and then became a negative value during the simulation (Fig. 3). These aforementioned results indicate that a normal trochlear groove and medial ridge can keep the patella in the appropriate position when the aLDFA is less than or equal to $102^{\circ}$. At aLDFAs exceeding this angle, the trochlea is no longer able to withstand the shear force generated by the quadriceps mechanism between the patella and trochlea. These findings could provide complementary information to clinical studies that reported good functional outcome of $87.5-93 \%$ and low recurrence rates of patellar luxation when DFO was applied in high-grade MPL patients with aLDFA of $>100^{\circ}-102^{\circ}$ $[9,11-14]$.

A reaction force of $0 \mathrm{kN}$ indicates patella luxation because the two elements, the femur and the patella, no

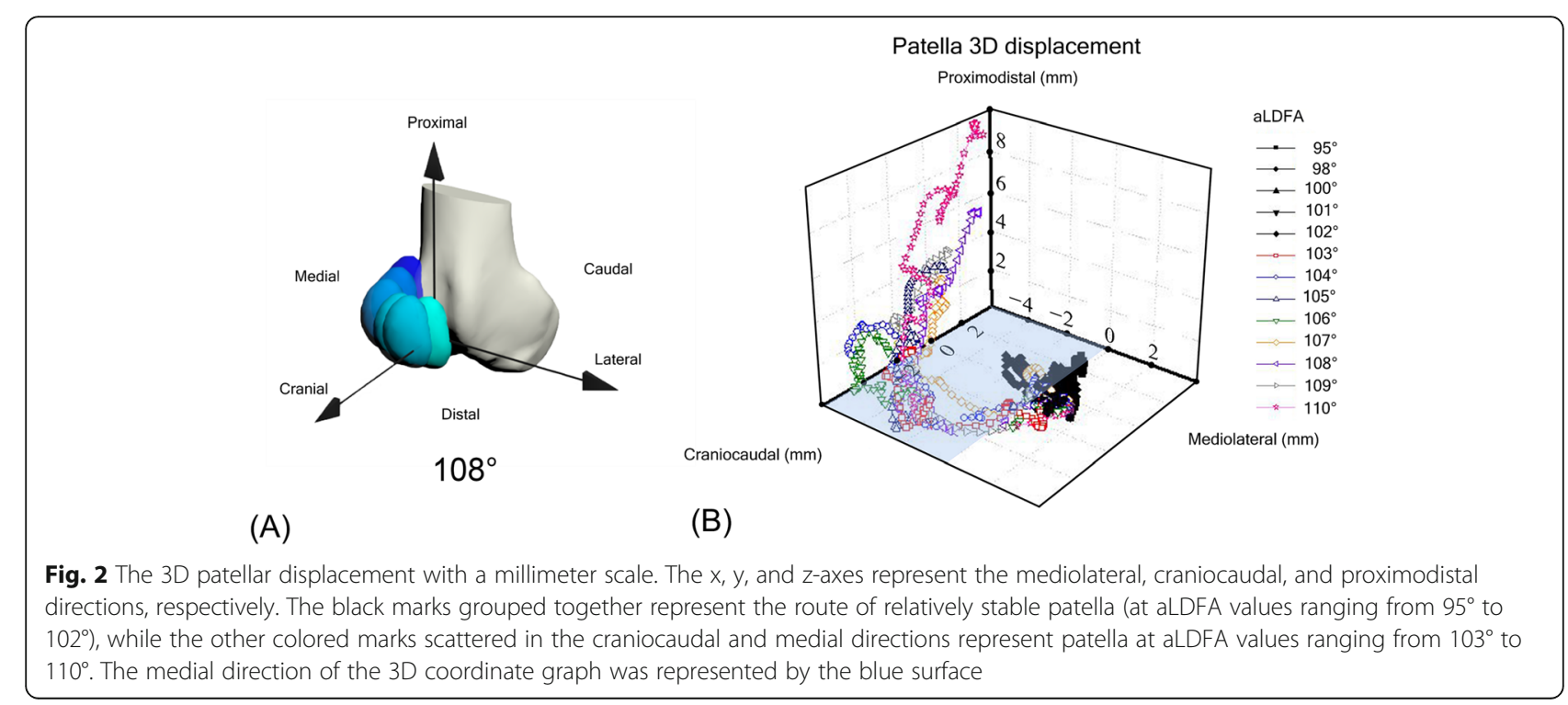




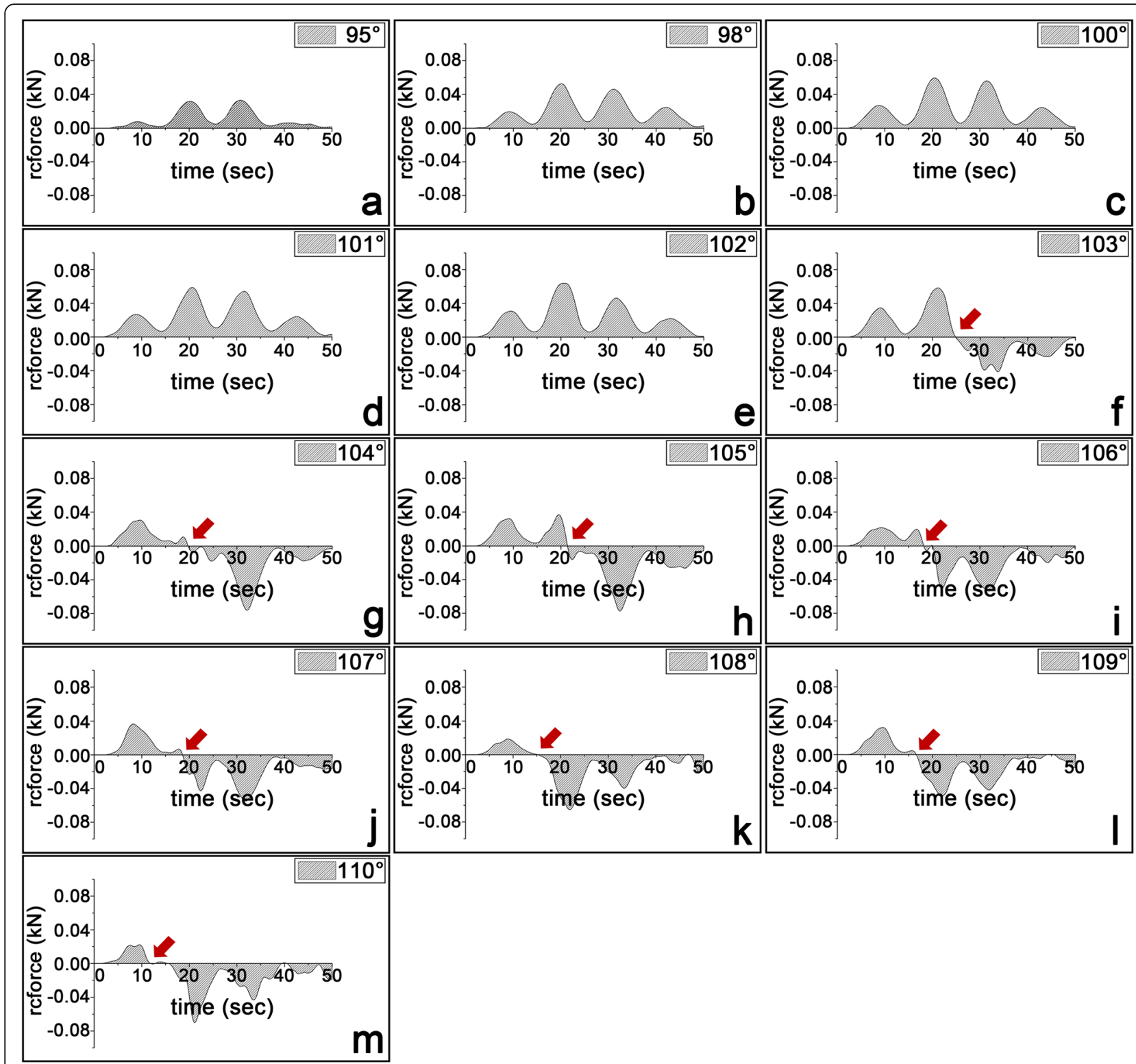

Fig. 3 The reaction force (rcforce) of the $x$-axis between patella and the femoral trochlear groove in the quadriceps-loading simulations. The Rcforce value reaches ' 0 ' and decreases to a negative value when the aLDFA is equal to and greater than $103^{\circ}$, respectively, and medial patella luxation occurs when the aLDFA is equal to and greater than $103^{\circ}$. Models corresponding to aLDFAs of $95^{\circ}(\mathbf{a}), 98^{\circ}(\mathbf{b}), 100^{\circ}(\mathbf{c}), 101^{\circ}(\mathbf{d}), 102^{\circ}(\mathbf{e})$, $103^{\circ}(\mathbf{f}), 104^{\circ}(\mathbf{g}), 105^{\circ}(\mathbf{h}), 106^{\circ}(\mathbf{i}), 107^{\circ}(\mathbf{j}), 108^{\circ}(\mathbf{k}), 109^{\circ}(\mathbf{I})$, and $110^{\circ}(\mathbf{m})$, and the rcforce units are kilonewtons $(1 \mathrm{kN}=1000 \mathrm{~N})$. The red arrow indicates when the rcforce is zero

longer react with each other. The negative rcforce was generated because the contact between the femur and the patella occurred in opposite directions. After luxation, the patella remained in lateral contact with the outside of the trochlear groove, and therefore, the rcforce was generated. However, since the patella was already luxated, this phenomenon was not clinically significant.

The total simulation time was set to 50 seconds because whether or not luxation occurred, time was required for the patellar motion to stabilize; thus, the time was set to 50 seconds to allow time for the motion of the patella to stabilize on all of the aLDFA models.

In terms of the outcome of luxation time, the trend was that the greater the aLDFA angle was, the shorter the time until luxation occurred, but some angles did not follow the trend consistently (Fig. 3). Patella luxation occurred at 19 seconds at $104^{\circ}$ and approximately 15 seconds at $108^{\circ}$, whereas luxation occurred at approximately 20 seconds at $105^{\circ}$ and $106^{\circ}$ and 16 seconds at $109^{\circ}$. At an aLDFA of $110^{\circ}$, temporary luxation occurred at 11 seconds, showing an unstable state, and 
complete luxation occurred at 15 seconds. In the reconstructed canine stifle model used in this study, there was no articular cartilage, synovial fluid, or other soft tissue to buffer the impact generated when the hard cortical bones of the patella and the trochlear groove collided with each other. Therefore, the above time discrepancies may have been due to the rebound phenomenon occurring as soon as the patella hit the medial ridge of the groove. Nevertheless, the fact that soft tissues were not modeled alone does not fully explain why the models with aLDFAs of $104^{\circ}$ and $108^{\circ}$ dislocated faster than those with other aLDFAs, and we could not determine the exact cause for this particular angle. In addition, patellar rotation, which is not possible with normal anatomy, is present after luxation, and the presence of muscles, ligaments, and the joint capsule prevent this rotation in vivo.

According to the normal aLDFA reported in large-breed dogs [27] and the aLDFA of MPL with a grade of less than 2 reported in small-breed dogs [10,28], angles of $95^{\circ}$ and $98^{\circ}$ were considered angles to be unaffected by varus deformities. Therefore, we assumed the critical angle that causes and increases the risk of patellar luxation to be $\geq 100^{\circ}$. However, we did not evaluate an aLDFA of $99^{\circ}$, which was within the range of values reported in grade 3 MPL cases in small-breeds, which was $100.53^{\circ} \pm 2.05^{\circ} .^{10}$ Although we can deduce the trend in patellar luxation as the varus angle changes, this point is a limitation of our study and needs to be carefully interpreted.

There are notable limitations of the present study. The FE model was not validated due to the lack of a comprehensive ex vivo testing model or motion analysis method to replicate the entire 3D biomechanics of the canine patellofemoral joint. Even though the quadriceps muscle was loaded during the experiment, the stifle angle was not extended further and remained static as the stance phase. Additionally, since the FE model was derived from an individual Beagle, differences in the stance posture and femoral joint angels according to breed conformation were not considered [27-29]. Moreover, the FE model did not include other factors affecting the MPL, such as cartilage, soft tissue stabilizers such as the components of the lateral aspect (vastus lateralis and lateral patellofemoral ligament), contracture of quadriceps muscle, femoral torsion, shapes of the femoral trochlea, and different positions of the tibial tuberosity. Thus, the results may not be generalizable to all dogs, and the calculated luxation risk may have been overestimated. Therefore, the aLDFA value in MPL obtained here should be considered as an indication of a trend caused by bony deformities and not as an absolute criterion. However, it is interesting that the results of this study are similar with anecdotal clinical recommendations for DFO in large-breed dogs with MPL $[9,13,14,30]$.

\section{Conclusions}

Overall, the results in the present simplified computer model show that distal femoral varus with an aLDFA equal to or greater than $103^{\circ}$ leads to MPL. The results based on an FE model are complementary to anecdotal cutoffs for DFO, although it should be applied to clinical patients with caution. The FE model of the canine stifle reported herein requires improvements to further analyze the effects of geometric features such as the breed size, bony shapes and soft tissues.

\section{Methods}

\section{Asymptomatic geometry preparation}

This study was approved by the National University of Chungnam's Institutional Animal Care and Use Committee (Number: CNU-01166). The CT images from a purpose-bred, adult, healthy, male laboratory Beagle dog (provided by the Animal Study Center of Chungnam National University) weighing $10.7 \mathrm{~kg}$ were used to create a femoral varus model and were selected as the baseline for the present study. The Beagle dog was released after all the experiment was conducted. No abnormalities were found via physical or orthopedic examinations or radiography in the Beagle dog. A computed tomographic (AlexionTM, Toshiba Medical Systems, Tochigi, Japan) scan of the Beagle dog was performed in the normal-standing position with the hip joint positioned according to that in the procedure previously reported [31]. The CT images were obtained using bone and soft tissue filters at $120 \mathrm{kV}$ and $120 \mathrm{~mA}$, respectively, and a slice thickness of $1 \mathrm{~mm}$. The aLDFA and anteversion angle (AA) of each femur of the Beagle dog were measured (both left and right aLDFAs were $95^{\circ}$ with an AA of $16^{\circ}$ ) (Fig. 4). The three-dimensional (3D) geometries of the left femur, patella, and tibia were extracted and segmented using 3D modeling software (Mimics innovation suite 20, Materialise NV, Leuven, Belgium). Fluoroscopic (BV Pulsera, Philips, Netherlands) images of the Beagle dog were taken while walking on the treadmill at $53 \mathrm{kVp}$ and $3.86 \mathrm{mAs}$ with a pulse rate of 30 frames/s and a pulse width of $11.1 \mathrm{~ms}$. The beam center of the fluoroscope was focused on the stifle mediolaterally. The geometries of these bones were manually aligned to the fluoroscopic images in the stance phase of gait cycle using previously described 3D-to2D image registration software (JointTrack, University of Florida, http://sourceforge.net/projects/jointtrack/) [32].

\section{Femoral varus model creation}

The asymptomatic normal stifle geometry of the Beagle and the center of rotation of angulation (CORA) were used to create the femoral varus geometry (Fig. 4). The position of CORA, located at the distal one-third of the femur, was based on the computed tomographic (CT) images of a client-owned Maltese dog's femur, which 


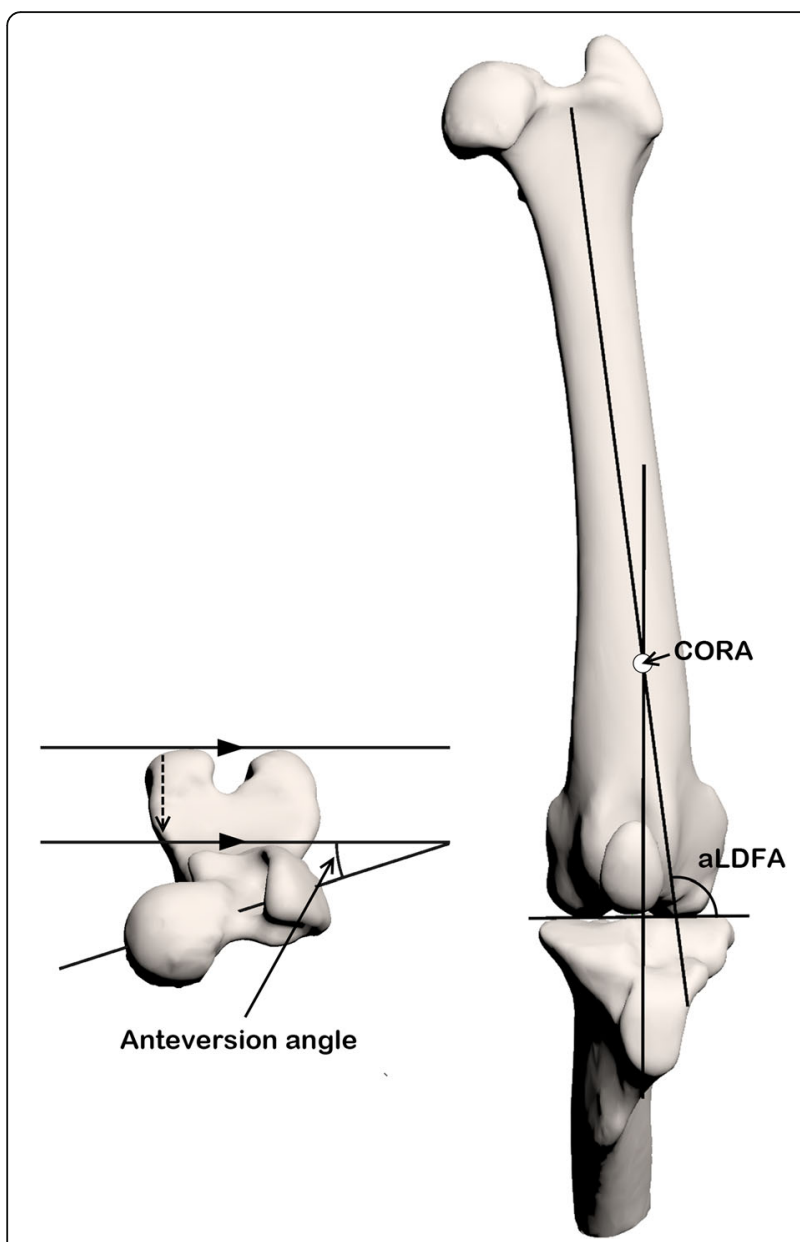

Fig. 4 Three-dimensional (3D) reconstructed model of the dog. The anteversion angle of the femur was $16^{\circ}$, and the aLDFA was $95^{\circ}$. The location of the CORA is indicated

had an aLDFA of $110^{\circ}$. It was consistent with a previous report of CORA in MPL $[8,9]$.

The normal femur geometry was then bent at the CORA using meshing software (3dsMax, Autodesk, San Rafael, CA, USA) to create deformed geometries. First, the geometry was scaled to fit the height of the clientowned Maltese dog's femur and bent at the same CORA level as that of the affected femur until it reached an aLDFA of $110^{\circ}$ [33]. After confirming that there was no translocation in the CORA position in the bent model, the remaining angle models were reproduced using a ratio of aLDFA of $95^{\circ}$ to $110^{\circ}$.

A total of thirteen different aLDFAs were simulated; normal aLDFA values from $95^{\circ}$ (baseline) to $98^{\circ}$ and affected angles from $100^{\circ}$ to $110^{\circ}$ were evaluated in onedegree increments.

The vertical position of the patellar segment was aligned to the stance phase of the stifle during the gait cycle based on the fluoroscopic images using the 3D-to$2 \mathrm{D}$ image registration technique. The angle between the normal femur and tibia segments in the sagittal plane was $127^{\circ}$ and remained static during the experiment. The ratio of the length of the patellar ligament to the length of the patella (L:P) was 1.55, which was within the range of values reported in clinically normal largebreed dogs [34]. During this process, the patella and the femoral trochlea remained anatomically undeformed so that every varus deformation had the same patella and trochlear groove geometries (Fig. 5).

\section{FE model development}

Thirteen stifle meshes were created using meshing software. To maintain an equivalent joint plane between the femur and tibia in all models, the tibia and distal epiphysis of the femur were fixed in the model before the femur was bent to create the deformity. Each stifle mesh model had specific tetrahedral solids because the solids were reconstructed by using a 3D stereolithography model before the mesh models were created (Fig. 5b). The mechanical behavior of the patellofemoral joint models was analyzed using Explicit FEM Multi Flexible Body dynamic analysis (LS-DYNA, Livermore Software Technology Corporation, Livermore, CA, USA). The cortical bone was modeled as a solid elastic material with a Young's modulus of $15.0 \mathrm{GPa}$ and a Poisson's ratio of 0.3 , which were chosen on the basis of the reported values [20, 24]. Cancellous bone was excluded due to its insignificant mechanical role in contact stress predictions, and bones were treated as complete solids in the model $[35,36]$.

The extensor or quadriceps mechanism includes the quadriceps (rectus femoris, vastus lateralis, vastus medialis, and vastus intermedius muscle), quadriceps tendon, patella, patellar ligament, and tibial tuberosity [2]. In the present study, only the rectus femoris muscle was modeled with a beam element with Hill's muscle model-based material (Material 156-MUSCLE), provided by LS-DYNA, to represent the quadriceps [37, 38]. The Hill-based model was designed according to Hill's equation describing the mechanical response of skeletal muscle [39]. In brief, Hill muscle models consist of a contractile element (CE) and parallel element (PE). The contractile element represents the force created by the activation of a muscle, while the parallel element represents the energy stored due to muscle elasticity. The total force $F_{M}$ is defined as the sum of the forces in the contractile element $\mathrm{F}_{\mathrm{CE}}$ and parallel element $\mathrm{F}_{\mathrm{PE}}$. The force generated by muscle activations $\left(\mathrm{F}_{\mathrm{CE}}\right)$ was calculated as follows:

$$
\mathrm{F}_{\mathrm{CE}}=a(t) \times F_{\max } \times f_{F L} \times f_{F V}
$$

where $a(t)$ is the activation level of the muscle, $F_{\max }$ is the peak isometric force, and $\mathrm{f}_{\mathrm{FL}}$ and $\mathrm{f}_{\mathrm{FV}}$ are normalized force-length and force-shortening velocity functions, 


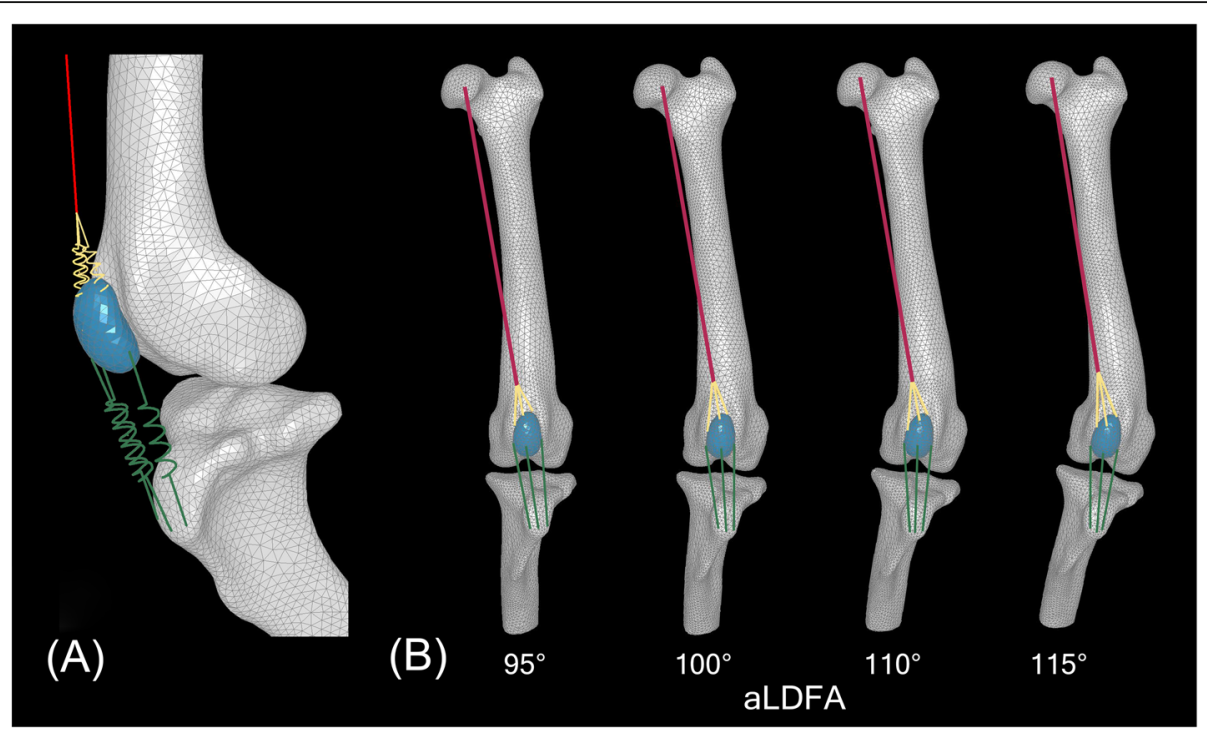

Fig. 5 a, A geometrically accurate FE model of the asymptomatic canine stifle, with an aLDFA of $95^{\circ}$. The FE model of the canine PF joint includes the rectus femoris muscle (red line), three functional elements of the quadriceps tendon (yellow springs) and the patellar ligament (green springs). The origin of the rectus femoris muscle in the model was set and fixed for all six degrees of motion at a virtual point matching the actual anatomical location of the ilium. $\mathbf{b}$, The geometries of four canine stifle models. The most distal part of the femur, including the patella and the trochlear groove, remained anatomically undeformed so that every varus deformation maintained the same geometry of the patella and the trochlear groove

respectively. In LS-DYNA, $\mathrm{F}_{\max }$ was calculated automatically using the peak isometric stress, physiological crosssectional area (PCSA) and $\mathrm{f}_{\mathrm{FL}}$ and $\mathrm{f}_{\mathrm{FV}}$ curves (Fig. 6). Thus, $\mathrm{F}_{\mathrm{CE}}$ can be simply calculated using the current length, shortening velocity, and activation level of the muscle.

The force generated by muscle elasticity $\left(\mathrm{F}_{\mathrm{PE}}\right)$ was calculated using the current length of muscle and an exponential relationship as follows:

$$
\mathrm{F}_{\mathrm{PE}}=\left\{\begin{array}{cc}
\epsilon \leq 0: & 0 \\
\epsilon>0: & \frac{1}{e^{k}-1}\left(e^{\frac{k}{L_{\max }}(L-1)}-1\right)
\end{array}\right.
$$

where $\epsilon$ is the strain of the muscle, $L$ is the current muscle length, $\mathrm{L}_{\max }$ is the maximum strain rate of muscle, and $\mathrm{k}$ is a dimensionless shape parameter controlling the rate of rise of the exponential function.

The origin of the rectus femoris muscle model was located based on the CT scan images with a sagittal plane hip angle of $125.2^{\circ}$ and constrained for all six degrees of freedom at a virtual point matching the actual anatomical location of the ilium (Fig. 5b).

To transfer more physiological load to the patella, the quadriceps tendon and ligament were separated into three beam elements and configured as linear spring materials with an elastic stiffness of $0.19 \mathrm{kN} / \mathrm{mm}[22$, $40,41]$. The properties of the materials used for the muscle were adapted from previous reports (Table 1) $[42,43]$.
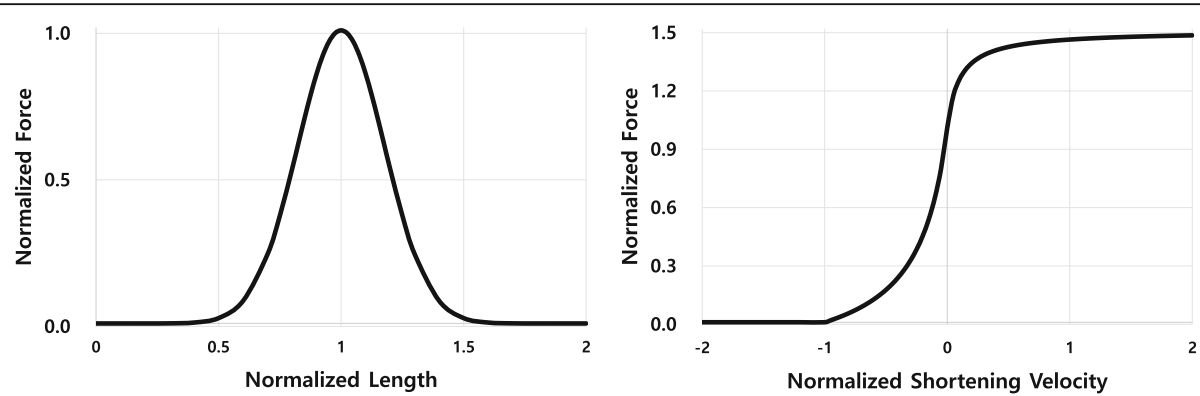

Fig. 6 Force-length and force-shortening velocity curves for rectus femoris muscle. The force-length and force-shortening velocity curves were used to calculate the coefficients of the muscle activation force using the initial and current relative length of the muscle, and the shortening velocity of the muscle, respectively 
Table 1 The properties of the parameters used to calculate the force generated by the rectus femoris

\begin{tabular}{lllllll}
\hline Parameter & Density & Initial relative length* & Maximum strain rate* & Peak isometric stress & Parallel constant $\mathbf{k}^{*}$ & PCSA \\
\hline Value & $1 \mathrm{e}-5 \mathrm{~kg} / \mathrm{mm}^{3}$ & 1.0 & 2.0 & $5 \mathrm{e}-4 \mathrm{GPa}$ & 6.0 & $1850 \mathrm{~mm}^{2}$ \\
\hline
\end{tabular}

*Note that parameters are normalized. For example, the peak isometric stress is normalized by dividing the peak isometric force by PCSA (physiological cross-sectional area)

\section{Boundary conditions}

Both the femur and tibia bone models were fully constrained to prevent unnecessary bony rotation or displacement during quadriceps loading, while the patella was unconstrained with three translational and three rotational degrees of freedom. The interaction between the patella and the femoral trochlea groove was defined as a surface-to-surface contact with a friction coefficient of $0.02[18,44]$.

Because a sudden increase in muscle-developed force can cause a large contact force between the patella and femur, which disarticulates the patella unnaturally, thirteen stifle models were simulated with a constant muscle activation level that linearly increased for 0 to $5 \mathrm{sec}$ from $0-20 \%$ and remained at $20 \%$ from time points 5 to 50 sec, without any changes in the stifle angle.

\section{Data Analysis}

Patellar positions and the reaction force between the patella and the femoral trochlear groove in all FE models were calculated under constant activation of the rectus femoris muscle. When muscle activation was initiated and the first contact point of the patella and the groove was zero, the patellar displacement was expressed as a negative value for the medial direction and a positive value for the lateral direction, according to the global coordinate value.

Then, the critical aLDFA causing medial luxation of the patella was determined. The reaction force (rcforce) $(\mathrm{kN})$ between the patella and the medial trochlear ridge was calculated for each aLDFA during the simulation. The total simulation time was set to 50 seconds to stabilize the motion of the patella in all of the aLDFA models.

\footnotetext{
Abbreviations

MPL: Medial patella luxation; aLDFAs: Anatomic lateral distal femoral angles; FE: Finite element; DFO: Distal femoral osteotomy; FVA: Femoral varus angle; FEM: Finite element method; Rcforce: Reaction force; IACUC: Institutional Animal Care and Use Committee; CORA: Center of rotation of angulation; CT: Computed tomography; AA: Anteversion angle; CE: Contractile element; SE: Serial element; PCSA: Physiologic cross sectional area
}

\section{Acknowledgements}

Not applicable.

\section{Authors' contributions}

HBL and JMJ conceived the study. JYL and HDS analyzed the data and drafted the manuscript. HDS and SJY conducted the computer modeling procedure and finite element analysis. SYK, SMJ, JMJ, and HBL assisted in designing the study, assisted with the data analysis and reviewed the manuscript. HBL provided general supervision. All authors read and approved the final manuscript.
Funding

Not applicable.

Availability of data and materials

The datasets used and/or analyzed during the current study are available from the corresponding author upon reasonable request.

Ethics approval and consent to participate

All procedures were approved by the National University of Chungnam's Institutional Animal Care and Use Committee (Number: CNU-01166). In-

formed written consent was provided by the owner of the Maltese dog.

Consent for publication

Not applicable.

Competing interests

The authors declare that they have no competing interests.

\section{Author details}

${ }^{1}$ Department of Veterinary Surgery, College of Veterinary Medicine, Chungnam National University, 34134 Daejeon, Republic of Korea.

${ }^{2}$ Department of Mechatronics Engineering, College of Engineering,

Chungnam National University, 34134 Daejeon, Republic of Korea.

${ }^{3}$ Department of Veterinary Clinical Sciences, College of Veterinary Medicine,

Purdue University, 47906 West Lafayette, IN, USA.

Received: 25 April 2020 Accepted: 26 October 2020

Published online: 03 December 2020

\section{References}

1. Roush JK. Canine patellar luxation. Vet Clin North Am Small Anim Pract. 1993;23:855-68.

2. DeCamp CE, Johnston SA, Dejardin LM, Schaefer SL. Brinker. Piermattei and Flo's Handbook of small animal orthopedics and fracture repair. 5th ed. St Louis: Elsevier; 2016. pp. 597-616.

3. Kowaleski MP, Boudrieau RJ, Pozzi A. Stifle joint. In: Johnston SA, Tobias KM, editors. Veterinary Surgery Small Animal. 2nd ed. St Louis: Elsevier Saunders; 2017. pp. 1141-4.

4. Bound N, Zakai D, Butterworth S, Pead M. The prevalence of canine patellar luxation in three centres. Vet Comp Orthop Traumatol. 2009;22:32-7.

5. Wangdee C, Theyse L, Techakumphu M, Soontornvipart K, Hazewinkel H. Evaluation of surgical treatment of medial patellar luxation in Pomeranian dogs. Vet Comp Orthop Traumatol. 2013;26:435-9.

6. Cashmore R, Havlicek M, Perkins N, et al. Major complications and risk factors associated with surgical correction of congenital medial patellar luxation in 124 dogs. Vet Comp Orthop Traumatol. 2014;27:263-70.

7. Arthurs GI, LANGLEY-HOBBS SJ. Complications associated with corrective surgery for patellar luxation in 109 dogs. Vet Surg. 2006;35:559-66.

8. Yasukawa S, Edamura K, Tanegashima K, et al. Evaluation of bone deformities of the femur, tibia, and patella in Toy Poodles with medial patellar luxation using computed tomography. Vet Comp Orthop Traumatol. 2016;29:29-38.

9. Brower BE, Kowaleski MP, Peruski AM, et al. Distal femoral lateral closing wedge osteotomy as a component of comprehensive treatment of medial patellar luxation and distal femoral varus in dogs. Vet Comp Orthop Traumatol. 2017;30:20-7.

10. Žilinčík M, Hluchý M, Takáč L, Ledecký V. Comparison of Radiographic Measurements of the Femur in Yorkshire Terriers with and without Medial Patellar Luxation. Vet Comp Orthop Traumatol. 2018;31:017-22.

11. Swiderski JK, Palmer RH. Long-term outcome of distal femoral osteotomy for treatment of combined distal femoral varus and medial patellar luxation: 12 cases (1999-2004). J Am Vet Med Assoc. 2007;231:1070-5. 
12. Hans EC, Kerwin SC, Elliott AC, Butler R, Saunders WB, Hulse DA. Outcome following surgical correction of grade 4 medial patellar luxation in dogs: 47 stifles (2001-2012). J Am Anim Hosp Assoc. 2016:52:162-9.

13. Roch $\mathrm{S}$, Gemmill T. Treatment of medial patellar luxation by femoral closing wedge ostectomy using a distal femoral plate in four dogs. J Small Anim Pract. 2008:49:152-8.

14. Dunlap AE, Kim SE, Lewis DD, Christopher SA, Pozzi A. Outcomes and complications following surgical correction of grade IV medial patellar luxation in dogs: 24 cases (2008-2014). J Am Vet Med Assoc. 2016;249:208-13.

15. Pfeiffer FM. The use of finite element analysis to enhance research and clinical practice in orthopedics. J Knee Surg. 2016;29:149-58.

16. Besier TF, Gold GE, Beaupré GS, Delp SL. A modeling framework to estimate patellofemoral joint cartilage stress in vivo. Med Sci Sports Exerc. 2005;37: 1924-30.

17. Halloran JP, Petrella AJ, Rullkoetter PJ. Explicit finite element modeling of total knee replacement mechanics. J Biomech. 2005;38:323-31.

18. Farrokhi S, Keyak J, Powers C. Individuals with patellofemoral pain exhibit greater patellofemoral joint stress: a finite element analysis study. Osteoarthritis Cartilage. 2011;19:287-94.

19. He C, He W, Li Y, et al. Biomechanics of Knee Joints after Anterior Cruciate Ligament Reconstruction. J Knee Surg. 2018;31:352-8.

20. Shahar R, Banks-Sills L, Eliasy R. Mechanics of the canine femur with two types of hip replacement stems. Vet Comp Orthop Traumatol. 2003;16:145-52.

21. Mazoochian F, Hölzer A, Jalali J, et al. Finite element analysis of the ovine hip: development, results and comparison with the human hip. Vet Comp Orthop Traumatol. 2012;25:301-6.

22. Fitzpatrick CK, Baldwin MA, Rullkoetter PJ. Computationally efficient finite element evaluation of natural patellofemoral mechanics. J Biomech Eng 2010;132:121013.

23. Fernandez J, Hunter P. An anatomically based patient-specific finite element model of patella articulation: towards a diagnostic tool. Biomech Model Mechanobiol. 2005:4:20-38

24. Shahar R, Banks-Sills L, Eliasy R. Stress and strain distribution in the intact canine femur: finite element analysis. Med Eng Phys. 2003;25:387-95.

25. McCartney W, MacDonald B, Ober CA, Lostado-Lorza R, Gómez FS. Pelvic modelling and the comparison between plate position for double pelvic osteotomy using artificial cancellous bone and finite element analysis. BMC Vet Res. 2018:14:100

26. de Albuquerque Bonelli M, Shah A, Goel V, Costa FS, da Costa RC. Development of a finite element model of the ligamentous cervica vertebral column of a Great Dane. Res Vet Sci. 2018;118:97-100.

27. Tomlinson J, Fox D, Cook JL, Keller GG. Measurement of femoral angles in four dog breeds. Vet Surg. 2007;36:593-8.

28. Phetkaew T, Kalpravidh M, Penchome R, Wangdee C. A Comparison of Angular Values of the Pelvic Limb with Normal and Medial Patellar Luxation Stifles in Chihuahua Dogs Using Radiography and Computed Tomography. Vet Comp Orthop Traumatol. 2018;31:114-23.

29. Sabanci SS, Ocal MK. Categorization of the pelvic limb standing posture in nine breeds of dogs. Anat Histol Embryol. 2018;47:58-63.

30. Olimpo M, Piras LA, Peirone B, Fox DB. Comparison of osteotomy technique and jig type in completion of distal femoral osteotomies for correction of medial patellar luxation. Vet Comp Orthop Traumatol. 2017;30:28-36.

31. Fujiki M, Misumi K, Sakamoto H. Laxity of canine hip joint in two positions with computed tomography. J Vet Med Sci. 2004;66:1003-6.

32. Kim SE, Jones SC, Lewis DD, et al. In-vivo three-dimensional knee kinematics during daily activities in dogs. J Orthop Res. 2015;33:1603-10.

33. Caouette C, Rauch F, Villemure I, et al. Biomechanical analysis of fracture risk associated with tibia deformity in children with osteogenesis imperfecta: a finite element analysis. J Musculosklet Neeuronal Interact. 2014;14:205-12.

34. Johnson AL, Probst CW, DeCamp CE, Rosenstein DS, Hauptman JG, Kern TL. Vertical position of the patella in the stifle joint of clinically normal largebreed dogs. Am J Vet Res. 2002;63:42-6.

35. Harris MD, Anderson AE, Henak CR, Ellis BJ, Peters CL, Weiss JA. Finite element prediction of cartilage contact stresses in normal human hips. J Orthop Res. 2012;30:1133-9.

36. Anderson AE, Ellis BJ, Maas SA, Peters CL, Weiss JA. Validation of finite element predictions of cartilage contact pressure in the human hip joint. J Biomech Eng. 2008;130:051008.
37. Mendes S, Silvestri C, Ray MH. Investigation of LS-DYNA ${ }^{\oplus}$ modeling for active muscle tissue. Proceedings of the 11th International LS-DYNA User Conference; 2010 June 6-8; Detroit, MI, USA: Livermore Software Technology Corp; 1996.

38. Kaiser S, Cornely D, Golder W, Garner M, Waibl H, Brunnberg L. Magnetic resonance measurements of the deviation of the angle of force generated by contraction of the quadriceps muscle in dogs with congenital patellar luxation. Vet Surg. 2001;30:552-8.

39. Mendes SB. The Development of an Improved Finite Element Muscle Model and the Investigation of the Pre-loading Effects of Active Muscle on the Femur During Frontal Crashes [master's thesis on the Internet]. Worcester, MA: Civil \& Environmental Engineering, Worcester Polytechnic Institute; 2010 [cited 2017 Mar 16]. Available from: https://digitalcommons.wpi.edu/etd-theses/1007.

40. Silvestri C, Mongiardini M, Ray MH, editors. Improvements and validation of an existing LS-DYNA model of the Knee-Thigh-Hip of a 50th percentile male including muscles and ligaments. Proceedings of the 7th European LSDYNA Conference; 2009 May 14-15; Salzburg, Austria: DYNAmore GmbH; 2009.

41. Fitzpatrick CK, Baldwin MA, Laz PJ, FitzPatrick DP, Lerner AL, Rullkoetter PJ. Development of a statistical shape model of the patellofemoral joint for investigating relationships between shape and function. J Biomech. 2011;44: 2446-52.

42. Shahar R, Milgram J. Morphometric and anatomic study of the hind limb of a dog. Am J Vet Res. 2001;62:928-33.

43. Munns SW, Jayaraman G, Luallin SR. Effects of pretwist on biomechanical properties of canine patellar tendon. Arthroscopy. 1994;10:404-11.

44. Besier TF, Gold GE, Delp SL, Fredericson M, Beaupré GS. The influence of femoral internal and external rotation on cartilage stresses within the patellofemoral joint. J Orthop Res. 2008;26:1627-35.

\section{Publisher's Note}

Springer Nature remains neutral with regard to jurisdictional claims in published maps and institutional affiliations.

Ready to submit your research? Choose BMC and benefit from:

- fast, convenient online submission

- thorough peer review by experienced researchers in your field

- rapid publication on acceptance

- support for research data, including large and complex data types

- gold Open Access which fosters wider collaboration and increased citations

- maximum visibility for your research: over $100 \mathrm{M}$ website views per year

At BMC, research is always in progress.

Learn more biomedcentral.com/submissions 